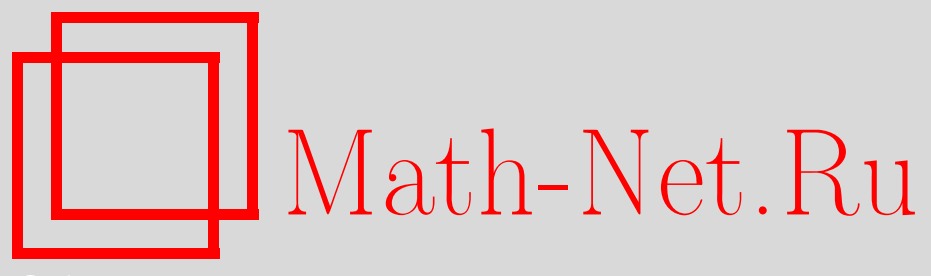

А. О. Игнатьев, О. А. Игнатьев, А. А. Солиман, Об асимптотической устойчивости и неустойчивости решений систем с импульсным воздействием, Матем. заметки, 2006, том 80, выпуск 4, 516-525

DOI: https://doi.org/10.4213/mzm2844

Использование Общероссийского математического портала Math-Net.Ru подразумевает, что вы прочитали и согласны с пользовательским соглашением http://www.mathnet.ru/rus/agreement

Параметры загрузки:

IP : 54.174 .149 .18

26 апреля 2023 г., 16:14:57

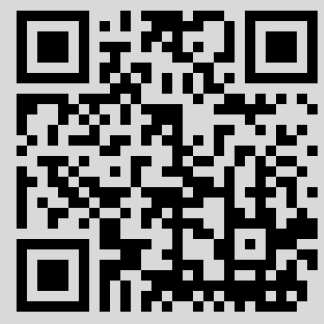




\section{ОБ АСИМПТОТИЧЕСКОЙ УСТОЙЧИВОСТИ И НЕУСТОЙЧИВОСТИ РЕШЕНИЙ СИСТЕМ С ИМПУЛЬСНЫМ ВОЗДЕЙСТВИЕМ}

\section{А. О. Игнатьев, О. А. Игнатьев, А. А. Солиман}

В работе изучается устойчивость нулевого решения системы обыкновенных дифференциальных уравнений с импульсным воздействием. С помощью метода функций Ляпунова получены критерии его асимптотической устойчивости и неустойчивости. Приведены иллюстративные примеры.

Библиография: 19 названий.

1. Введение. При математическом описании эволюции реальных процессов с кратковременными возмущениями во многих случаях длительностью возмущений удобно пренебречь и считать, что эти возмущения носят “мгновенный" характер. Такая идеализация приводит к необходимости исследовать динамические системы с разрывными траекториями или, иначе, дифференциальные уравнения с импульсным воздействием. K первым работам в этом направлении следует отнести [1], [2]. Итогом первых работ по дифференциальным уравнениям с импульсным воздействием явилась монография [3], в которой изложены основы этой теории. В последние годы заметно увеличилось число математических работ по исследованию различных аспектов теории импульсных систем [4]-[16], что вызвано запросами новейшей техники. Настоящая статья посвящена изучению устойчивости решений систем с импульсным воздействием. Она является продолжением и развитием работ [17], [18].

2. Определения и некоторые предварительные результаты. Рассмотрим систему обыкновенных дифференциальных уравнений с импульсным воздействием

$$
\begin{aligned}
\frac{d x}{d t} & =f(t, x), & & t \neq \tau_{k}, \\
\Delta x & =I_{k}(x), & t & =\tau_{k},
\end{aligned}
$$

где $t \in \mathbb{R}_{+}:=[0, \infty)$ - время, $k \in \mathbb{N}\left(\mathbb{N}\right.$ - это множество натуральных чисел), $\tau_{k}-$ константы, $x \in \mathbb{R}^{n}, f: \mathbb{R}^{n+1} \rightarrow \mathbb{R}^{n}, I_{k}: \mathbb{R}^{n} \rightarrow \mathbb{R}^{n}$. Уравнения (2.1) описывают динамику системы, состоящей из двух частей: непрерывной (при $t \neq \tau_{k}$ ), описываемой обыкновенными дифференциальными уравнениями, и дискретной (в моменты $\tau_{k}$ ), когда решения системы получают скачкообразные изменения. Обозначим

$$
B_{H}:=\left\{x \in \mathbb{R}^{n}:\|x\|=\sqrt{x_{1}^{2}+\cdots+x_{n}^{2}}<H\right\}
$$

(C) А. О. ИГнатьев, О.А. ИГнатьев, А. А. Солиман, 2006 


$$
G_{k}:=\left\{(t, x) \in \mathbb{R}^{n+1}: \tau_{k-1}<t<\tau_{k}, x \in B_{H}\right\}, \quad G:=\bigcup_{k=1}^{\infty} G_{k} .
$$

Сформулируем гипотезы $(\Gamma 1)-(\Gamma 5)$, которым может удовлетворять система (2.1).

ГипотезА $(\Gamma 1)$. Функция $f=\left(f_{1}, \ldots, f_{n}\right): G \rightarrow \mathbb{R}^{n}$ непрерывна в каждом из множеств $G_{k}, k \in \mathbb{N} ; f(t, 0) \equiv 0$, и существует константа $L>0$ такая, что $\| f(t, x)-$ $f(t, y)\|\leqslant L\| x-y \|$ при $(t, x) \in G,(t, y) \in G, x \in B_{H}, y \in B_{H}$.

ГипотезА (Г2). Функции $I_{k}: B_{H} \rightarrow \mathbb{R}^{n}, k \in \mathbb{N}$, непрерывны в $B_{H}$ и $I_{k}(0)=0$ при $k \in \mathbb{N}$.

ГиПотезА (ГЗ). Существует константа $h \in(0, H)$ такая, что если $x \in B_{h}$, то $x+I_{k}(x) \in B_{H}$ при $k \in \mathbb{N}$.

ГипотеЗА (Г4). Константы $\tau_{k}$ удовлетворяют условиям

$$
0=\tau_{0}<\tau_{1}<\tau_{2}<\cdots, \quad \lim _{k \rightarrow \infty} \tau_{k}=\infty
$$

ГиПотезА (Г5). Константы $\tau_{k}$ удовлетворяют условию: для любых $T>0, t>0$ отрезок $[t, t+T]$ содержит не более $p$ констант $\tau_{k}$, причем число $p$ зависит только от $T$ и не зависит от $t$.

Будем обозначать $x\left(t, t_{0}, x_{0}\right)$ при $t>t_{0}$ решение системы $(2.1)$, удовлетворяющее условию $x\left(t_{0}, t_{0}, x_{0}\right)=x_{0}$ в случае, если $t_{0} \neq \tau_{k}, k \in \mathbb{N}$. Если же $t_{0}=\tau_{k}$ при какомлибо натуральном $k$, то под выражением $x\left(t, t_{0}, x_{0}\right)$ будем понимать $x\left(t, t_{0}+0, x_{0}+\right.$ $\left.I_{k}\left(x_{0}\right)\right)$ (при $\left.t>t_{0}\right)$.

При выполнении гипотез (Г1)-(Г3) система (2.1) допускает тривиальное решение

$$
x \equiv 0 .
$$

ОПРЕДЕЛЕНиЕ 2.1. Тривиальное решение системы (2.1) называется устойчивым, если для любых $\varepsilon>0, t_{0} \in \mathbb{R}_{+}$можно указать $\delta=\delta\left(\varepsilon, t_{0}\right)>0$ такое, что если $\left\|x_{0}\right\|<\delta$, то $\left\|x\left(t, t_{0}, x_{0}\right)\right\|<\varepsilon$ при $t>t_{0}$.

ОПРЕДЕЛЕНИЕ 2.2. Решение (2.2) системы (2.1) называется притягивающим, если для любого $t_{0} \in \mathbb{R}_{+}$существует $\eta=\eta\left(t_{0}\right)>0$ и для любых $\varepsilon>0$ и $x_{0} \in B_{\eta}$ существует $\sigma=\sigma\left(\varepsilon, t_{0}, x_{0}\right)>0$ такое, что $\left\|x\left(t, t_{0}, x_{0}\right)\right\|<\varepsilon$ для всех $t \geqslant t_{0}+\sigma$.

ОПРЕДЕЛЕНИЕ 2.3. Тривиальное решение системы (2.1) называется асимптотически устойчивым, если оно устойчиво и притягивающее.

ОПРЕДЕЛЕНиЕ 2.4. Будем говорить, что функция $\omega: \mathbb{R}_{+} \rightarrow \mathbb{R}_{+}$принадлежит $\kappa л а с с у \mathscr{K}(\omega \in \mathscr{K})$, если она непрерывна, строго возрастает и $\omega(0)=0$.

Следуя [4], введем следующие определения.

ОПРЕДЕЛЕНИЕ 2.5. Будем говорить, что функция $V: \mathbb{R}_{+} \times B_{H} \rightarrow \mathbb{R}$ принадлежит классу $\mathscr{V}_{0}\left(V \in \mathscr{V}_{0}\right)$, если $V$ непрерывна на любом из множеств $G_{k}, V(t, 0) \equiv 0$ для $t \in \mathbb{R}_{+}$, существуют конечные пределы

$$
V\left(t_{0}-0, x_{0}\right)=\lim _{\substack{(t, x) \rightarrow\left(t_{0}, x_{0}\right) \\(t, x) \in G_{k}}} V(t, x), \quad V\left(t_{0}+0, x_{0}\right)=\lim _{\substack{(t, x) \rightarrow\left(t_{0}, x_{0}\right) \\(t, x) \in G_{k+1}}} V(t, x),
$$

и справедливо равенство $V\left(t_{0}-0, x_{0}\right)=V\left(t_{0}, x_{0}\right)$. 
Пусть $f(t, x)$ является $C^{j-1}$-функцией: $f: G \rightarrow \mathbb{R}^{n}$, а $V$ является $C^{j}$-функцией $V: \mathbb{R}_{+} \times \mathbb{R}^{n} \rightarrow \mathbb{R}$. Определим $V_{s}: \mathbb{R}_{+} \times B_{H} \rightarrow \mathbb{R}^{n}:$

$$
V_{s}(t, x):=\frac{\partial V_{s-1}}{\partial t}+\sum_{i=1}^{n} \frac{\partial V_{s-1}}{\partial x_{i}} f_{i}(t, x), \quad s=1,2, \ldots, j,
$$

при $t \neq \tau_{k}$ и $V_{s}\left(\tau_{k}, x\right)=V_{s}\left(\tau_{k}-0, x\right)$, где $V_{0}(t, x):=V(t, x)$. В частности,

$$
V_{1}(t, x)=\left.\frac{d V}{d x}\right|_{(2.1)}=\frac{\partial V}{\partial t}+\sum_{i=1}^{n} \frac{\partial V}{\partial x_{i}} f_{i}(t, x)
$$

ОПредЕЛЕНиЕ 2.6. Будем говорить, что функция $V: \mathbb{R}_{+} \times B_{H} \rightarrow \mathbb{R}$ принадлежит классу $\mathscr{V}_{m}\left(V \in \mathscr{V}_{m}\right)$, если $V$ является $m$ раз непрерывно-дифференцируемой на любом из множеств $G_{k}$ и существуют конечные пределы

$$
V_{r}\left(t_{0}-0, x_{0}\right)=\lim _{\substack{(t, x) \rightarrow\left(t_{0}, x_{0}\right) \\(t, x) \in G_{k}}} V_{r}(t, x), \quad V_{r}\left(t_{0}+0, x_{0}\right)=\lim _{\substack{(t, x) \rightarrow\left(t_{0}, x_{0}\right) \\(t, x) \in G_{k+1}}} V_{r}(t, x), \quad r=0,1, \ldots, m .
$$

Здесь $V_{0}=V$.

В работах [10], [18] показано, что если система (2.1) такова, что выполнены гипотезы $(\Gamma 1)-(\Gamma 4)$ и существуют функции $V \in \mathscr{V}_{1}, a, c \in \mathscr{K}$ такие, что

$$
\begin{gathered}
V(t, x) \geqslant a(\|x\|) \quad \text { при } \quad(t, x) \in \mathbb{R}_{+} \times B_{H}, \\
\left.\frac{d V}{d t}\right|_{(2.1)} \leqslant-c(\|x\|) \quad \text { при } \quad(t, x) \in G, \\
V\left(\tau_{k}+0, I_{k}(x)+x\right)-V\left(\tau_{k}, x\right) \leqslant 0 \quad \text { при } \quad k \in \mathbb{N},
\end{gathered}
$$

то решение (2.2) системы (2.1) асимптотически устойчиво.

Целью настоящей работы является получение более мягких условий асимптотической устойчивости в предположении, что функция $V$ удовлетворяет условию $d V / d t \leqslant 0$ вместо (2.3).

В дальнейшем нам потребуется следующая

Лемма 2.1. Пусть $h(t)$ - скалярная функиия, имеющая точки разрыва первого рода при

$$
t=b_{1}, \quad t=b_{2}, \quad \ldots, \quad \text { əде } 0<b_{1}<b_{2}<\cdots, \quad \lim _{i \rightarrow \infty} b_{i}=\infty,
$$

причем $h\left(b_{i}-0\right)=h\left(b_{i}\right)$, в каждом из интервалов $\left(b_{i}, b_{i+1}\right)$ бункиия $h(t)$ является $j+1$ раз непрерывно-дифферениируемой, ее производные $h^{\prime}(t), h^{\prime \prime}(t), \ldots, h^{(j+1)}$ ограничены при $t \in \bigcup_{i=1}^{\infty}\left(b_{i}, b_{i+1}\right]$, и выполняется условие: константы $b_{i}$ удовлетворяют гипотезе (Г5) и выполнены предельные соотношения

$$
\lim _{i \rightarrow \infty}\left[h\left(b_{i}+0\right)-h\left(b_{i}-0\right)\right]=0, \quad \lim _{i \rightarrow \infty}\left[h^{(r)}\left(b_{i}+0\right)-h^{(r)}\left(b_{i}-0\right)\right]=0, \quad r=1, \ldots, j .
$$

Если

$$
\lim _{t \rightarrow \infty} h(t)=0,
$$

mo

$$
\lim _{t \rightarrow \infty} h^{(r)}(t)=0, \quad r=1,2, \ldots, j .
$$


ЗАмЕчАНИЕ 2.1. Под производными любого порядка в точках $b_{i}$ будем понимать левосторонние производные.

ДокАзАТЕЛЬСтво лЕммы. Покажем вначале, что

$$
\lim _{t \rightarrow \infty} h^{\prime}(t)=0 \text {. }
$$

Предположим противное: пусть существуют $\xi>0$ и моменты времени $T_{m} \in \mathbb{R}_{+}$, $m \in \mathbb{N}$, такие, что $T_{m} \rightarrow \infty$ при $m \rightarrow \infty$ и $\left|h^{\prime}\left(T_{m}\right)\right| \geqslant 2 \xi$. Это означает, что либо $h^{\prime}\left(T_{m}\right) \geqslant 2 \xi$, либо $h^{\prime}\left(T_{m}\right) \leqslant-2 \xi$. Из ограниченности $h^{\prime \prime}(t)$ и условия

$$
\lim _{i \rightarrow \infty}\left[h^{\prime}\left(b_{i}+0\right)-h^{\prime}\left(b_{i}-0\right)\right]=0
$$

следует, что существуют $\zeta>0$ и $M_{1} \in \mathbb{N}$ такие, что

$$
h^{\prime}(t) \geqslant \frac{3}{2} \xi
$$

или

$$
h^{\prime}(t) \leqslant-\frac{3}{2} \xi
$$

при $t \in\left[T_{m}-\zeta, T_{m}+\zeta\right], m \geqslant M_{1}$. Отсюда, воспользовавшись условиями леммы, получаем, что существует $M_{2} \in \mathbb{N}, M_{2} \geqslant M_{1}$, такое, что при $m \geqslant M_{2}$ выполняется условие

$$
h\left(T_{m}+\zeta\right) \geqslant h\left(T_{m}\right)+\xi \zeta
$$

в случае (2.6) или условие

$$
h\left(T_{m}+\zeta\right) \leqslant h\left(T_{m}\right)-\xi \zeta
$$

в случае (2.7). Из предельного соотношения (2.4) следует, что существует такое $M \geqslant T_{M_{2}}>0$, что $|h(t)|<\xi \zeta / 2$ при $t \geqslant M$. С другой стороны, из неравенств (2.8), (2.9) следует справедливость неревенств

$$
\left|h\left(T_{m}+\zeta\right)\right| \geqslant \frac{1}{2} \xi \zeta \quad \text { при } \quad m \in \mathbb{N}, \quad T_{m} \geqslant M .
$$

Но эти неравенства противоречат предположению (2.4). Полученное противоречие доказывает справедливость предельного соотношения (2.5).

Аналогично можно показать, что

$$
\lim _{t \rightarrow \infty} h^{\prime \prime}(t)=0, \quad \ldots, \quad \lim _{t \rightarrow \infty} h^{(j)}(t)=0 .
$$

Лемма доказана.

\section{3. Основные результаты.}

Теорема 3.1. Пусть система (2.1) такова, что $f \in C^{j}$ при $(t, x) \in G$, выполняются гипотезы (Г1)-(Г4) и существуют $V \in \mathscr{V}_{j+1}, w_{1} \in \mathscr{K}, w_{2} \in \mathscr{K}$ такие, что

(A) $V(t, x) \geqslant w_{1}(\|x\|) n p u(t, x) \in \mathbb{R}_{+} \times B_{H}$;

(Б) $V_{1}(t, x)=d V / d t \leqslant 0 n p u(t, x) \in G$; 
(B) $V_{s}(t, x), s=1, \ldots, j+1$, ограничены при $(t, x) \in G$;

(Г) $\sum_{s=1}^{j} V_{s}^{2}(t, x) \geqslant w_{2}(\|x\|)$;

(Д) $V\left(\tau_{k}+0, x+I_{k}(x)\right)-V\left(\tau_{k}, x\right) \leqslant 0$ при $x \in B_{H}, k \in \mathbb{N}$;

(Е) константы $\tau_{k}$ удовлетворяют гипотезе (Г5) и справедливы предельные соотношения

$$
\lim _{k \rightarrow \infty}\left[V_{r}\left(\tau_{k}+0, x+I_{k}(x)\right)-V_{r}\left(\tau_{k}, x\right)\right]=0, \quad r=0,1, \ldots, j,
$$

равномерно по $x \in B_{H}$.

Тогда решение (2.2) системы (2.1) асимптотически устойчиво.

ДокАЗАтЕЛЬСтво. Из условий $(\mathrm{A}),($ Б), (Д) в силу [18] следует, что решение (2.2) системы (2.1) устойчиво. Возьмем произвольные $\varepsilon \in(0, H), t_{0} \in \mathbb{R}_{+}$и $x_{0} \in B_{\delta}$, где $\delta>0$ таково, что $\left\|x\left(t, t_{0}, x_{0}\right)\right\|<\varepsilon$ при $t>t_{0}$. Обозначим $v(t)=V\left(t, x\left(t, t_{0}, x_{0}\right)\right)^{1}$. Функция $v(t)$ положительна и не возрастает, следовательно, предел $\lim _{t \rightarrow \infty} v(t)$ существует и он неотрицателен:

$$
\lim _{t \rightarrow \infty} v(t)=\eta \geqslant 0
$$

Покажем, что

$$
\lim _{t \rightarrow \infty} v_{1}(t)=0
$$

где $v_{1}(t)=V_{1}\left(t, x\left(t, t_{0}, x_{0}\right)\right)$. Предположим противное, т.е. будем считать, что предельное соотношение (3.2) не выполняется. Тогда существуют $\beta>0$ и последовательность $\left\{T_{i}\right\}_{i=1}^{\infty}\left(T_{i} \in \mathbb{R}_{+}, i \in \mathbb{N}, T_{i+1}>T_{i}, T_{i} \rightarrow \infty\right.$ при $\left.i \rightarrow \infty\right)$ такая, что $v_{1}\left(T_{i}\right) \leqslant-2 \beta<0$. Согласно условиям (A), (Б), (В), (Е) теоремы существуют $\gamma>0$ и $M_{1} \in \mathbb{N}$ такие, что $v_{1}(t) \leqslant-\beta$ при $t \in\left[T_{i}, T_{i}+\gamma\right], i \geqslant M_{1}$. Из условий (Б), (Д) следует, что

$$
v\left(T_{i}+\gamma\right) \leqslant v\left(T_{i}\right)-\beta \gamma
$$

при $i \geqslant M_{1}$. Так как

$$
\lim _{i \rightarrow \infty} v\left(T_{i}\right)=\eta
$$

то отсюда и из (3.3) следует

$$
\lim _{i \rightarrow \infty} v\left(T_{i}+\gamma\right) \leqslant \eta-\beta \gamma
$$

Но так как $T_{i}+\gamma \rightarrow \infty$ при $i \rightarrow \infty$, то полученное неравенство противоречит предельному соотношению (3.1). Полученное противоречие доказывает справедливость равенства (3.2).

Используя предельное соотношение (3.2) и лемму 2.1, получаем, что

$$
\lim _{t \rightarrow \infty} \sum_{s=1}^{j} v_{s}^{2}(t)=0,
$$

где $v_{s}(t)=V_{s}\left(t, x\left(t, t_{0}, x_{0}\right)\right)$. Покажем теперь, что нулевое решение системы $(2.1)$ является притягивающим. Для этого нужно показать, что

$$
\lim _{t \rightarrow \infty}\left\|x\left(t, t_{0}, x_{0}\right)\right\|=0 .
$$

\footnotetext{
${ }^{1}$ Под $v\left(\tau_{k}\right)$ по-прежнему будем понимать $v\left(\tau_{k}-0\right)$.
} 
Предположим противное: пусть существуют $\alpha>0$ и последовательность $\left\{t_{i}\right\}(i \in \mathbb{N}$, $t_{i} \rightarrow \infty$ при $\left.i \rightarrow \infty\right)$ такие, что $\left\|x\left(t_{i}, t_{0}, x_{0}\right)\right\| \geqslant \alpha$. Из условия (Г) следует, что

$$
\sum_{s=1}^{j} v_{s}^{2}\left(t_{i}\right) \geqslant w_{2}\left(\left\|x\left(t_{i}, t_{0}, x_{0}\right)\right\|\right) \geqslant w_{2}(\alpha)>0 .
$$

Полученное неравенство противоречит (3.4), откуда следует справедливость предельного соотношения (3.5). Теорема доказана.

ЗАмечаниЕ 3.1. Если в доказанной теореме положить $j=1, I_{k}(x) \equiv 0, k \in \mathbb{N}$, $x \in B_{H}$, то получаем известную теорему Марачкова [19].

Обозначим

$$
Q_{h}:=\left\{(t, x) \in \mathbb{R}_{+} \times B_{h}: V(t, x)>0\right\} .
$$

Теорема 3.2. Предположим, что система (2.1) такова, что $f \in C^{j} n р и(t, x) \in$ $G$, выполняются гипотезы (Г1)-(Г4) и существуют функиии $V \in \mathscr{V}_{j+1} u w \in \mathscr{K}$ maкuе, что

(а) для любых $t \in \mathbb{R}_{+}, \varepsilon \in(0, H)$ множество $Q_{\varepsilon}$ непусто;

(б) $V(t, x), V_{1}(t, x), \ldots, V_{j+1}(t, x)$ ограничены в $Q_{H}$;

(в) $d V / d t=V_{1}(t, x) \geqslant 0$ в $Q_{H}$;

(г) $\sum_{s=1}^{j} V_{s}^{2}(t, x) \geqslant w(V(t, x))$;

(д) $\left.\Delta V\right|_{t=\tau_{k}}=V\left(\tau_{k}+0, x+I_{k}(x)\right)-V\left(\tau_{k}, x\right) \geqslant 0$ nрu $x \in B_{H}, k \in \mathbb{N}$;

(е) выполняется условие (Е) теоремы 3.1 .

Тогда решение (2.2) системы (2.1) неустойчиво.

ДоказАтельство. Выберем произвольные $\varepsilon \in(0, H), t_{0} \in \mathbb{R}_{+}$, и пусть $\delta$ - сколь угодно малое положительное число. Покажем, что существуют $x_{0} \in B_{\delta}$ и $t_{1}>t_{0}$ такие, что $\left\|x\left(t_{1}, t_{0}, x_{0}\right)\right\| \geqslant \varepsilon$. Выберем $x_{0} \in B_{\delta}$ таким образом, что $V\left(t_{0}, x_{0}\right)>0$. Такой выбор возможен в силу условия (a).

Предположим противное: при любом $t>t_{0}$ выполняется неравенство

$$
\left\|x\left(t, t_{0}, x_{0}\right)\right\|<\varepsilon,
$$

откуда следует, что $(t, x) \in Q_{\varepsilon}$ и функция $V\left(t, x\left(t, t_{0}, x_{0}\right)\right)$ ограничена. Из условий (в), (д) имеем

$$
v(t)=V\left(t, x\left(t, t_{0}, x_{0}\right)\right) \geqslant V\left(t_{0}, x_{0}\right)=\beta>0 .
$$

Функция $v(t)$ ограничена и неубывает, следовательно, существует предел

$$
\lim _{t \rightarrow \infty} v(t)=\eta \geqslant \beta>0 .
$$

Аналогично тому, как это сделано при доказательстве теоремы 3.1, можно показать, чTо

$$
\lim _{t \rightarrow \infty} v_{s}(t)=0, \quad s=1, \ldots, j,
$$

где $v_{s}=V_{s}\left(t, x\left(t, t_{0}, x_{0}\right)\right)$, откуда получаем, что имеет место предельное соотношение (3.4). Из условия (г) следует, что

$$
\sum_{s=1}^{j} v_{s}^{2}(t)=\sum_{s=1}^{j} V_{s}^{2}\left(t, x\left(t, t_{0}, x_{0}\right)\right) \geqslant w\left(V\left(t, x\left(t, t_{0}, x_{0}\right)\right) \geqslant w(\beta)>0\right.
$$


при $t \geqslant t_{0}$. Однако соотношения (3.4) и (3.7) противоречат друг другу. Полученное противоречие показывает, что предположение (3.6) неверно, т.е. решение (2.2) системы (2.1) неустойчиво.

ПримеР 3.1. Рассмотрим систему дифференциальных уравнений с импульсным воздействием

$$
\begin{aligned}
\frac{d x}{d t} & =-y-a(t) x, \quad \frac{d y}{d t}=x, \quad t \neq \tau_{k}, k=1,2, \ldots, \\
\left.\Delta x\right|_{t=\tau_{k}} & =-\frac{x}{k},\left.\quad \Delta y\right|_{t=\tau_{k}}=0,
\end{aligned}
$$

где $a(t)=2-\cos 2 \pi t-\cos \left(2 \pi \log _{2}(t+1)\right), \tau_{k}=3 k, k \in \mathbb{N}$. Система (3.8) допускает тривиальное решение

$$
x=0, \quad y=0 .
$$

Для исследования устойчивости этого решения воспользуемся функцией Ляпунова

$$
V=\frac{1}{2}\left(x^{2}+y^{2}\right)
$$

Находим последовательно

$$
\begin{aligned}
& V_{1}=\frac{d V}{d t}=-a(t) x^{2}, \quad V_{2}=\frac{d V_{1}}{d t}=\left(2 a^{2}-a^{\prime}\right) x^{2}+2 a x y, \\
& V_{3}=\frac{d V_{2}}{d t}=\left(6 a a^{\prime}-a^{\prime \prime}-4 a^{3}+2 a\right) x^{2}+\left(4 a^{\prime}-6 a^{2}\right) x y-2 a y^{2}, \\
& V_{4}=\frac{d V_{3}}{d t}=\left(6 a^{\prime 2}+8 a a^{\prime \prime}-a^{\prime \prime \prime}-24 a^{2} a^{\prime}+6 a^{\prime}+8 a^{4}-10 a^{2}\right) x^{2} \\
& +\left(-28 a a^{\prime}+6 a^{\prime \prime}+14 a^{3}-8 a\right) x y+\left(6 a^{2}-6 a^{\prime}\right) y^{2}, \\
& V_{5}=\frac{d V_{4}}{d t}=\left(20 a^{\prime} a^{\prime \prime}+10 a a^{\prime \prime \prime}-a^{\prime \prime \prime \prime}-60 a a^{2}-40 a^{2} a^{\prime \prime}+12 a^{\prime \prime}\right. \\
& \left.+80 a^{3} a^{\prime}-60 a a^{\prime}-16 a^{5}+34 a^{3}-8 a\right) x^{2} \\
& +\left(-40 a^{\prime 2}-50 a a^{\prime \prime}+8 a^{\prime \prime \prime}+118 a^{2} a^{\prime}-32 a^{\prime}-30 a^{4}+40 a^{2}\right) x y \\
& +\left(40 a a^{\prime}-12 a^{\prime \prime}-14 a^{3}+8 a\right) y^{2} .
\end{aligned}
$$

Производные функции $a(t)$ имеют следующий вид:

$$
a^{(s)}(t)=\alpha_{s}(t)+\beta_{s} \frac{\sin \left(2 \pi \log _{2}(t+1)\right)}{(t+1)^{s}}+\gamma_{s} \frac{\cos \left(2 \pi \log _{2}(t+1)\right)}{(t+1)^{s}},
$$

где $\beta_{s}, \gamma_{s}, s \in \mathbb{N},-$ это константы, причем $\beta_{1}=2 \pi / \ln 2, \gamma_{1}=0$,

$$
\alpha_{1}=2 \pi \sin 2 \pi t, \quad \alpha_{2}=(2 \pi)^{2} \cos 2 \pi t, \quad \alpha_{3}=-(2 \pi)^{3} \sin 2 \pi t, \quad \alpha_{4}=-(2 \pi)^{4} \cos 2 \pi t .
$$

Функция $a(t)$ неотрицательна, причем $a(t)=0$ при $t=t_{n}=2^{n}-1(n=0,1,2, \ldots)$.

Рассмотрим функцию $W=V_{1}^{2}+V_{2}^{2}+V_{3}^{2}+V_{4}^{2}+V_{5}^{2}$. Покажем, что $W(t, x, y)-$ функция определенно-положительная относительно $x$ и $y$. Покажем вначале, что функция $W\left(t_{n}, x, y\right)$ определенно-положительна. Учитывая $a\left(t_{n}\right)=0, a^{\prime}\left(t_{n}\right)=0$, находим последовательно:

$$
\begin{aligned}
& V_{1}\left(t_{n}, x, y\right)=0, \quad V_{2}\left(t_{n}, x, y\right)=0, \quad V_{3}\left(t_{n}, x, y\right)=-a^{\prime \prime}\left(t_{n}\right) x^{2}, \\
& V_{4}\left(t_{n}, x, y\right)=-a^{\prime \prime \prime}\left(t_{n}\right) x^{2}+6 a^{\prime \prime}\left(t_{n}\right) x y, \\
& V_{5}\left(t_{n}, x, y\right)=\left(-a^{\prime \prime \prime \prime}\left(t_{n}\right)+12 a^{\prime \prime}\left(t_{n}\right)\right) x^{2}+8 a^{\prime \prime \prime}\left(t_{n}\right) x y-12 a^{\prime \prime}\left(t_{n}\right) y^{2} .
\end{aligned}
$$


Заметим, что $\gamma_{2}=(2 \pi)^{2} / \ln ^{2} 2>0$, откуда получаем, что

$$
a^{\prime \prime}\left(t_{n}\right)>(2 \pi)^{2} .
$$

Используя (3.10), получаем

$$
W\left(t_{n}, x, y\right) \geqslant V_{3}^{2}\left(t_{n}, x, y\right)+V_{5}^{2}\left(t_{n}, x, y\right) \geqslant(2 \pi)^{4} x^{4}+V_{5}^{2}\left(t_{n}, x, y\right) .
$$

Учитывая, что $V_{5}\left(t_{n}, 0, y\right)=-12 a^{\prime \prime}\left(t_{n}\right) y^{2}$, и используя неравенство $(3.10)$, можно сделать вывод, что $W\left(t_{n}, x, y\right)>2 w\left(x^{2}+y^{2}\right)$, где $w \in \mathscr{K}$. Функции $a(t)$ и $a^{(s)}(t)$, $s=1,2,3,4$, являются равномерно непрерывными при $t \in \mathbb{R}_{+}$, поэтому существует $\varepsilon>0$ такое, что

$$
W(t, x, y) \geqslant w\left(x^{2}+y^{2}\right)
$$

при $t_{n}-\varepsilon \leqslant t \leqslant t_{n}+\varepsilon$. Покажем, что при

$$
t \notin\left[t_{n}-\varepsilon, t_{n}+\varepsilon\right]
$$

функция $W$ также удовлетворяет свойству (3.11) при любом $n \in \mathbb{N}$. Заметим, что при условии (3.12) существует $\omega>0$ такое, что $a(t)>\omega$. Оценим $W$ при таких $t$ :

$$
\begin{aligned}
\left.W(t, x, y)\right|_{t \notin\left[t_{n}-\varepsilon, t_{n}+\varepsilon\right]} & \geqslant\left.\left(V_{1}^{2}+V_{3}^{2}\right)\right|_{t \notin\left[t_{n}-\varepsilon, t_{n}+\varepsilon\right]} \geqslant \psi(t, x, y) \\
& =\omega^{2} x^{4}+\left[\left(6 a a^{\prime}-a^{\prime \prime}-4 a^{3}+2 a\right) x^{2}+\left(4 a^{\prime}-6 a^{2}\right) x y-2 a y^{2}\right]^{2} .
\end{aligned}
$$

Найдем $\psi(t, x, y)$ при $x=0$ :

$$
\psi(t, 0, y)=4 a^{2}(t) y^{4}>4 \omega^{2} y^{4} .
$$

Отсюда, учитывая, что $a(t), a^{\prime}(t), a^{\prime \prime}(t)$ - функции, ограниченные при $t \in \mathbb{R}_{+}$, получаем, что при $t \notin\left[t_{n}-\varepsilon, t_{n}+\varepsilon\right]$ функция $W$ положительно определена относительно $x, y$. Следовательно, существует $w \in \mathscr{K}$ такая, что выполняется неравенство (3.11) при $t \in \mathbb{R}_{+},(x, y) \in \mathbb{R}^{2}$. Найдем теперь

$$
\left.\Delta V\right|_{t=\tau_{k}}=\frac{1}{2}\left[\left(x+\left.\Delta x\right|_{t=\tau_{k}}\right)^{2}+\left(y+\left.\Delta y\right|_{t=\tau_{k}}\right)^{2}\right]-\frac{1}{2}\left(x^{2}+y^{2}\right)=-\frac{x^{2}}{k}\left(1-\frac{1}{2 k}\right) \leqslant 0 .
$$

Заметим, что $V_{1}, V_{2}, V_{3}, V_{4}, V_{5}, V_{6}:=d V_{5} / d t$ можно представить в виде

$$
V_{m}=F_{m}(t) x^{2}+P_{m}(t) x y+Q_{m}(t) y^{2}, \quad m=1,2,3,4,5,6,
$$

где $F_{m}, P_{m}, Q_{m}$ - ограниченные непрерывные функции $t$. Следовательно, эти функции ограничены при $x^{2}+y^{2}<H^{2}$, и

$$
\begin{gathered}
\left.\Delta V_{m}\right|_{t=\tau_{k}}=F_{m}\left(\tau_{k}\right)\left[\left(x+\left.\Delta x\right|_{t=\tau_{k}}\right)^{2}-x^{2}\right]+P_{m}\left(\tau_{k}\right)\left[\left(x+\left.\Delta x\right|_{t=\tau_{k}}\right)\left(y+\left.\Delta y\right|_{t=\tau_{k}}\right)-x y\right] \\
+Q_{m}\left(\tau_{k}\right)\left[\left(y+\left.\Delta y\right|_{t=\tau_{k}}\right)^{2}-y^{2}\right]=-F_{m}\left(\tau_{k}\right) \frac{x^{2}}{k}\left(1-\frac{1}{2 k}\right)-P_{m}\left(\tau_{k}\right) \frac{x y}{k}
\end{gathered}
$$

откуда получаем $\left.\lim _{k \rightarrow \infty} \Delta V_{m}\right|_{t=\tau_{k}}=0, m=1, \ldots, 5$.

Таким образом, условия (А)-(Д), (E1) теоремы 3.1 выполнены, и можно утверждать, что решение (3.9) системы (3.8) асимптотически устойчиво. 
ПримеР 3.2. Рассмотрим систему обыкновенных дифференциальных уравнений с импульсным воздействием

$$
\begin{gathered}
\frac{d x}{d t}=-y+a(t) x, \quad \frac{d y}{d t}=x, \quad t \neq \tau_{k}, \quad k=1,2, \ldots, \\
\left.\Delta x\right|_{t=\tau_{k}}=\frac{x}{k},\left.\quad \Delta y\right|_{t=\tau_{k}}=0
\end{gathered}
$$

где $a(t)$ такая же, как в предыдущем примере. Система (3.13) допускает нулевое решение (3.9). Покажем, что оно неустойчиво. Рассмотрим функцию

$$
V=\frac{1}{2}\left(x^{2}+y^{2}\right)
$$

Находим

$$
\frac{d V}{d t}=a(t) x^{2} \geqslant 0,\left.\quad \Delta V\right|_{t=\tau_{k}}=\frac{x^{2}}{k}+\frac{x^{2}}{2 k^{2}} \geqslant 0 .
$$

Аналогично предыдущему примеру можно показать, что существует такая $w \in \mathscr{K}$, что $W=V_{1}^{2}+V_{2}^{2}+V_{3}^{2}+V_{4}^{2}+V_{5}^{2} \geqslant w\left(x^{2}+y^{2}\right)$. То есть все условия теоремы 3.2 выполнены, следовательно, нулевое решение системы (3.13) неустойчиво.

\section{СПИСОК ЦИТИРОВАННОЙ ЛИТЕРАТУРЫ}

[1] В. Д. Мильман, А. Д. Мышкис, "Об устойчивости движения при наличии толчков", Сиб. матем. ж., 1:2 (1960), 233-237.

[2] А. Д. Мышкис, А. М. Самойленко, "Системы с толчками в заданные моменты времени", Матем. сб., 74:2 (1967), 202-208.

[3] А. М. Самойленко, Н. А. Перестюк, Дифференциальные уравнения с импульсным воздействием, Вища школа, Киев, 1987.

[4] D. D. Bainov, P. S. Simeonov, Systems with Impulse Effect: Stability, Theory and Applications, Halsted Press, New York-Chichester-Brisbane-Toronto, 1989.

[5] V. Lakshmikantham, D. D. Bainov, P. S. Simeonov, Theory of Impulsive Differential Equations, World Scientific, Singapore-New Jersey-London, 1989.

[6] V. Lakshmikantham, X. Liu, "On quasi stability for impulsive differential systems", Nonlinear Analysis, 13:7 (1989), 819-829.

[7] A. Cabada, E. Liz, "Discontinuous impulsive differential equations with nonlinear boundary conditions", Nonlinear Analysis, 28:9 (1997), 1491-1497.

[8] Р. И. Гладилина, А. О. Игнатьев, "О необходимых и достаточных условиях асимптотической устойчивости для импульсных систем”, Укр. матем. ж., 55:8 (2003), 10351043.

[9] Р. И. Гладилина, А. О. Игнатьев, “Об устойчивости периодических систем с импульсным воздействием", Матем. заметки, 76:1 (2004), 44-51.

[10] А.О. Игнатьев, "Метод функций Ляпунова в задачах устойчивости решений систем дифференциальных уравнений с импульсным воздействием", Матем. сб., 194:10 (2003), 117-132.

[11] S. D. Borysenko, G. Iovane, P. Giordano, "Investigations of the properties motion for essential nonlinear systems perturbed by impulses on some hypersurfaces", Nonlinear Anal., 62 (2005), 345-363.

[12] А. А. Бойчук, Н. А. Перестюк, А. М. Самойленко, "Периодические решения импульсных дифференциальных систем в критических случаях", Дифферени. уравнения, 27:9 (1991), 1516-1521. 
[13] A. A. Soliman, "Stability criteria of impulsive differential systems", Appl. Math. Comput., 134 (2003), 445-457.

[14] S. Hu, V. Lakshmikantham, "Periodic boundary value problems for second order impulsive differential systems", Nonlinear Analysis, 13:1 (1989), 75-87.

[15] K. K. Kenzhebaev, A. N. Stanzhitskii, "Invariant sets of impulsive systems and their stability", Nonlinear Oscillations, 7:1 (2004), 78-82.

[16] A. A. Soliman, "On cone perturbing Liapunov function for impulsive differential systems", Appl. Math. Comput., 163 (2005), 1069-1071.

[17] A. M. Samoilenko, N. A. Perestyuk, Impulsive Differential Equations, World Scientific, Singapore-New Jersey-London, 1995.

[18] С. И. Гургула, Н. А. Перестюк, "Об устойчивости положения равновесия импульсных систем”, Матем. физика, 31 (1982), 9-14.

[19] В. П. Марачков, "Об одной теореме устойчивости”, Изв. физ.-матем. общ-ва и научноисслед. ин-та матем. и механ. при Казанском ун-те. Сер. 3, 12 (1940), 171-174.

\section{А. О. Игнатьев}

Институт прикладной математики и механики НАН Украины, Донецк, Украина

E-mail: mila@budinf.donetsk.ua, aoignat@mail.ru, ignat@iamm.ac.donetsk.ua

\section{О. А. Игнатьев}

Kent State University, Kent, Ohio, USA

E-mail: aignatye@kent.edu

\section{А. А. Солиман (А. А. Soliman)}

Benha University, Kalubia, Egypt

E-mail: a-a_soliman@hotmail.com
Поступило 26.07.2005

Исправленный вариант 08.02.2006 